

\title{
Normal Findings on Pretreatment Transcranial Ultrasound in Patients Treated with Sonothrombolysis
}

\author{
Elias Johansson ${ }^{\mathrm{a}, \mathrm{b}}$ Thomas $\mathrm{Gu}^{\mathrm{a}}$ Per Wester ${ }^{\mathrm{b}}$ \\ Departments of a Pharmacology and Clinical Neuroscience and ${ }^{\mathrm{b}}$ Public Health and Clinical \\ Medicine, Umeå University, Umeå, Sweden
}

\section{Key Words}

Sonothrombolysis · Stroke · Thrombolysis · Thrombolysis in Brain Ischemia grade ·

Transcranial ultrasound

\begin{abstract}
In populations with a high $(\geq 14)$ median National Institute of Health Stroke Score (NIHSS), a normal finding of Thrombolysis in Brain Ischemia grade 5 (TIBI 5) in the artery of interest has been reported to be an unusual finding when transcranial ultrasound is performed during thrombolysis. In such instances, a stroke mimic can be suspected, but there are alternative pathophysiological explanations. In this case series, the median NIHSS was relatively low (5), and $33 \%(6 / 18)$ of the patients treated with thrombolysis had TIBI 5 in the artery of interest at the time of treatment initiation. These 6 patients had normal findings on the computerized tomography angiography. Only $33 \%(2 / 6)$ of these patients were stroke mimics, the remaining had either lacunar $(n=2)$ or cortical strokes $(n=2)$. These cortical stroke patients probably had a pretreatment recanalization marked by partial symptom regression before treatment onset. Compared to patients with TIBI $<5$ at baseline, the patients with TIBI 5 at baseline tended to be younger ( $p=0.19$, Mann-Whitney test) and more often have lacunar syndrome $(p=$ $0.18, \chi^{2}$ test). Thus, among patients treated with thrombolysis and with a low median NIHSS, a finding of TIBI 5 is not unusual. This does not mean that the patient has a stroke mimic per se, and it tends to be more common among patients with lacunar syndrome than among patients with cortical syndromes.

(C) 2014 S. Karger AG, Basel
\end{abstract}




\section{Introduction}

Transcranial ultrasound has several important indications in vascular neurology, one of these being sonothrombolysis $[1,2]$. Sonothrombolysis is a therapy for acute ischemic stroke in which continuous transcranial ultrasound applied to the artery occlusion is added to intravenous thrombolysis. The degree of artery occlusion can be classified according to Thrombolysis in Brain Ischemia (TIBI) grades, where $0-3$ is an occlusive or subocclusive lesion, 4 is a stenotic lesion, and 5 is normal [3].

In observational studies of patients who received thrombolysis, only $6-17 \%$ had normal findings (TIBI 5) in the middle cerebral artery (MCA) [4, 5]. However, in our initial experience of sonothrombolysis, many of the patients treated with thrombolysis had TIBI 5 in the MCA. Since much of what we know about sonothrombolysis is based on randomized trials, and TIBI 0-3 in the MCA was required for inclusion in these trials [6], much remains unknown about patients who receive thrombolysis and have TIBI 5 at treatment onset. TIBI 5 is an expected finding in acute settings when there is no artery occlusion in the examined artery, such as in stroke mimics, if recanalization has already occurred, and if a nonaffected artery is examined (e.g., when the symptoms of a basilar occlusion mimic an MCA occlusion). Although distal branch occlusion can be detected as TIBI 3 proximal to the occlusion [7], very distal branch occlusions and lacunar strokes might be identified as TIBI 5 in acute settings; however, this has not been thoroughly explored.

The aim of our study was to present a few cases of patients treated with thrombolysis and who had TIBI 5 at treatment onset. We also examined possible explanations for our findings.

\section{Materials and Methods}

The Umeå Stroke Center at the University Hospital of Northern Sweden provides primary stroke care for a local population of 140,000. In 2012, we treated 379 patients with stroke and 151 patients with TIA. Fifty-eight patients were treated with intravenous thrombolysis (11\% of all stroke and TIA patients). The door-to-needle time was $<30 \mathrm{~min}$ in $36(62 \%)$ patients. Intravenously administered alteplase $(0.9 \mathrm{mg} / \mathrm{kg}$, maximum dose $90 \mathrm{mg}$ ) was used, with $10 \%$ given as a bolus and $90 \%$ infused over $1 \mathrm{~h}$.

Beginning in September 2011, we investigated and treated all patients eligible for thrombolysis with sonothrombolysis (intravenous thrombolysis combined with continuous ultrasound) instead of intravenous thrombolysis alone whenever possible. We reproduced the methods of the treatment arm in the Combined Lysis of Thrombus in Brain Ischemia Using Transcranial Ultrasound and Systemic tPA (CLOTBUST) study [3], except treatment was given up to $4.5 \mathrm{~h}$ after symptom onset and irrespective of the National Institutes of Health Stroke Score (NIHSS), as long as there were clear-cut focal neurological symptoms that warranted treatment. A standardized fast-track protocol [5] was used except that the ophthalmic artery was not examined because time between the beginning of the examination and initiation of thrombolysis treatment was short. In accordance with this protocol, the MCA, anterior cerebral artery, and posterior cerebral artery (PCA) were examined, with emphasis on the MCA. The MCA was examined as distally as possible, especially when no other pathology was detected, in order to exclude a detectable distal occlusion. A ST3 $2 \mathrm{MHz}$ Power M-Mode transcranial Doppler (Spencer Technologies) was used. Two ultrasound operators (E.J. and T.G.) performed all examinations; both operators had passed the TIBI grade examination used in the CLOTBUST study. The ultrasound examination and continuous monitoring were always initiated immediately before or $<10$ min after the bolus injection of tissue plasminogen activator (tPA). Continuous monitoring of the contralateral artery was initiated as soon as possible after the ipsilateral side.

We classified the patients according to Trial of Org 10172 in Acute Stroke Treatment (TOAST) criteria [8]. We compared patients with small vessel disease etiology (lacunar syndrome) with the remaining patients (cortical syndrome). 
Statistics

Continuous variables are given as median with interquartile range (IQR). We compared groups with the $\chi^{2}$ and Mann-Whitney tests. We defined a statistically significant finding as $p<0.05$ and a statistical tendency as $\mathrm{p}<0.20$. We used SPSS 20.0 as statistical software.

The introduction of sonothrombolysis at our clinic was monitored in a quality assurance study. The local ethical committee was consulted and stated that this study did not require ethics approval.

\section{Results}

Treatment with sonothrombolysis was attempted in about $30 \%$ of the patients who received thrombolysis. In the remaining cases, no ultrasound operator was available. Until April 15, 2013, treatment with sonothrombolysis was attempted in 23 patients. Of these 23 patients, 5 were excluded due to a poor acoustic window $(n=4)$ or brain stem stroke $(n=1)$. Thus, 18 patients treated with thrombolysis and with a sufficient acoustic window were included. Of these, 16 (89\%) were treated with sonothrombolysis and 2 (11\%) with thrombolysis alone at the discretion of the ultrasound operator according to TIBI 5 findings.

The median age was 71 years (IQR 61-78), and 61\% of the patients were male. Fifteen had MCA territory symptoms, 1 had a PCA infarction (with TIBI 3 in the corresponding PCA), and 2 had stroke mimics. Among 16 nonmimic cases, the median NIHSS at baseline was 5 (IQR $3-7) ; 6 \%(1 / 16)$ had scores of $>10,63 \%(10 / 16)$ had scores of $5-10$, and $31 \%(5 / 16)$ had scores of $2-4$. In the 16 nonmimic cases, the TOAST classification revealed lacunar syndrome (small vessel disease) in 4 (25\%) cases and cortical syndrome in 12 (75\%) cases (cardioembolism, $n=7$; undetermined because of no detected etiology, $n=4$, and undetermined because of concurrent large vessel disease and cardioembolism, $n=1$ ). The case with large vessel disease was a symptomatic carotid occlusion; there were no patients with intracranial stenosis. The 4 patients with lacunar syndrome had a similar NIHSS (range 6-8), whereas the 12 patients with cortical syndrome had a more diverse NIHSS (range 2-11). At baseline, 5 (28\%) patients had TIBI 2, 7 (39\%) patients had TIBI 3, and 6 (33\%) patients had TIBI 5. Of the 12 patients with TIBI 2 or 3 at baseline, 5 (42\%) achieved complete recanalization (i.e., changed to TIBI 5) and 1 (8\%) achieved partial recanalization (i.e., improved $\geq 1$ TIBI grade, but did not reach TIBI 5). All patients underwent another computed tomography (CT) or magnetic resonance imaging (MRI) scan after $24 \mathrm{~h}$, and none had a symptomatic intracerebral hemorrhage. Bridging therapy with intra-arterial thrombectomy was initiated in 1 patient, but the affected artery had recanalized at the time when the cerebral angiogram was performed.

The prevalence of TIBI 5 was 33\% ( $n=6 ; 95 \%$ CI 9-57). The 6 patients with TIBI 5 at baseline are presented in detail in table 1 , and the spectral curves of the 4 cases with TIBI 5 that had a stroke (not a stroke mimic) are displayed in figure 1 . All 6 cases had normal findings on the acute CT angiography. The 4 patients with stroke all had new infarctions on CT/MRI at $24 \mathrm{~h}$, and the localization corresponded with their clinical diagnosis of lacunar or cortical syndrome. The patients with cortical syndrome had either cardioembolic stroke $(n=1)$ or undetermined etiology because of no detected etiology $(n=1)$; both cases with cortical syndrome had partial symptom regression before treatment initiation. We compared the patients with TIBI 5 and TIBI $<5$ on pretreatment ultrasound (table 2). Although no statistically significant differences were detected, the TIBI 5 patients tended to be younger $(p=0.19$, Mann-Whitney test) and more often have lacunar syndrome ( $p=0.18, \chi^{2}$ test). 
Johansson et al.: Normal Findings on Pretreatment Transcranial Ultrasound in Patients

Treated with Sonothrombolysis

Table 1. Case presentation of the 6 patients with TIBI 5 at pretreatment ultrasound

\begin{tabular}{|c|c|c|c|c|c|c|c|}
\hline Nc & $\begin{array}{l}\text { o. Age, } \\
\text { sex }\end{array}$ & Presentation & $\begin{array}{l}\text { NIHSS before } \\
\text { treatment/ } \\
\text { at discharge }\end{array}$ & $\begin{array}{l}\text { Acute CT and } \\
\text { acute } C T A^{\mathrm{a}}\end{array}$ & $\begin{array}{l}\text { Treatment } \\
\text { and OTT }\end{array}$ & $\mathrm{CT} / \mathrm{MRI}$ at $24 \mathrm{~h}$ & Comments \\
\hline 1 & $66, \mathrm{M}$ & $\begin{array}{l}\text { Lacunar syndrome: left- } \\
\text { sided hemihypestesia and } \\
\text { hemiparesis, slight } \\
\text { dysarthria, no cortical } \\
\text { symptoms }\end{array}$ & $7 / 7$ & $\begin{array}{l}\text { CT: normal } \\
\text { CTA: normal }\end{array}$ & $\begin{array}{l}\text { SonoT } \\
60 \mathrm{~min}\end{array}$ & $\begin{array}{l}\text { CT: new infarction } \\
\text { in the internal } \\
\text { capsule and corona } \\
\text { radiata }\end{array}$ & - \\
\hline 2 & $59, \mathrm{M}$ & $\begin{array}{l}\text { Lacunar syndrome: right- } \\
\text { sided hemihypestesia and } \\
\text { hemiparesis, no cortical } \\
\text { symptoms }\end{array}$ & $8 / 0$ & $\begin{array}{l}\text { CT: two old right- } \\
\text { sided lacunar infarcts } \\
\text { CTA: normal }\end{array}$ & $\begin{array}{l}\text { SonoT } \\
64 \text { min }\end{array}$ & $\begin{array}{l}\text { CT: new small } \\
\text { infarction in the left } \\
\text { internal capsule }\end{array}$ & $\begin{array}{l}\text { Temporary (10 min) } \\
\text { right arm paresis } \\
\text { during tPA infusion, } \\
\text { MCA flow was } \\
\text { unchanged }\end{array}$ \\
\hline 3 & $41, \mathrm{~F}$ & $\begin{array}{l}\text { Cortical syndrome: right- } \\
\text { sided hemihypestesia and } \\
\text { hemiparesis, slight aphasia }\end{array}$ & $4 / 0^{\mathrm{b}}$ & $\begin{array}{l}\text { CT: normal } \\
\text { CTA: normal }\end{array}$ & $\begin{array}{l}\text { SonoT } \\
69 \mathrm{~min}\end{array}$ & $\begin{array}{l}\text { MRI: } 2 \text { new small } \\
\text { left-sided cortical } \\
\text { infarctions }\end{array}$ & $\begin{array}{l}\text { Significant } \\
\text { improvement before } \\
\text { treatment was } \\
\text { initiated }\end{array}$ \\
\hline 4 & $81, \mathrm{M}$ & $\begin{array}{l}\text { Cortical syndrome: total } \\
\text { expressive and slight } \\
\text { impressive aphasia, slight } \\
\text { paresis of the right arm and } \\
\text { leg }\end{array}$ & $3 / 3$ & $\begin{array}{l}\text { CT: old cortical } \\
\text { ischemia in the left } \\
\text { cortex and cerebellum } \\
\text { CTA: normal }\end{array}$ & $\begin{array}{l}\text { T alone } \\
172 \text { min }\end{array}$ & $\begin{array}{l}\text { CT: new } 3 \mathrm{~cm} \\
\text { infarction, left side, } \\
\text { at the Brocas area } \\
\text { and motor cortex }\end{array}$ & $\begin{array}{l}\text { Slight improvement } \\
\text { before treatment was } \\
\text { initiated }\end{array}$ \\
\hline 5 & $76, \mathrm{M}$ & $\begin{array}{l}\text { Stroke mimic: left-sided } \\
\text { hemiparesis, gaze deviation } \\
\text { to the right, two seizures }\end{array}$ & NA/NA & $\begin{array}{l}\text { CT: normal } \\
\text { CTA: normal }\end{array}$ & $\begin{array}{l}\text { Sonot } \\
\text { NA }\end{array}$ & CT: no new findings & Postictal paresis \\
\hline 6 & $37, \mathrm{~F}$ & $\begin{array}{l}\text { Stroke mimic: left-sided } \\
\text { hemiparesis, blurred vision, } \\
\text { chest pain, dyspnea }\end{array}$ & NA/NA & $\begin{array}{l}\text { CT: normal } \\
\text { CTA: normal }\end{array}$ & $\begin{array}{l}\text { T alone } \\
\text { NA }\end{array}$ & CT: no new findings & Functional symptoms \\
\hline
\end{tabular}

CTA = CT angiography of the neck and brain arteries; OTT $=$ onset to treatment; Sono $\mathrm{T}=$ sonothrombolysis; $\mathrm{T}$ alone $=$ thrombolysis without continuous ultrasound; NA = no data available.

${ }^{a}$ Of the neck arteries and intracerebral arteries. ${ }^{b}$ Slight palsy of the right hand remained at discharge, but this was not sufficiently pronounced to warrant an NIHSS point for right arm palsy.

\section{Discussion}

The main findings in this study were that in a population treated with sonothrombolysis and a low median NIHSS, normal findings (TIBI 5) of the presumed affected artery are not unusual. The finding of TIBI 5 does not mean that the patient has a stroke mimic per se, and it tends to be more common among patients with lacunar syndrome than among patients with cortical syndrome.

Transcranial ultrasound has a satisfactory good agreement with both CT angiography and digital subtraction angiography $[9,10]$. In this study, the results of the CT angiography confirmed the findings of the transcranial ultrasound: in all 6 TIBI 5 cases with TIBI 5, the CT angiography had the same finding (no detectable artery occlusion).

In two previous studies of consecutive patients treated with thrombolysis, the median NIHSS was 14 and 17.5, with a prevalence of TIBI 5 of 6 and 17\%, respectively [3,4]. One of these studies reported a median NIHSS of about 10 in the TIBI 5 group [4]. In the present study, the median NIHSS was lower (5) and the prevalence of TIBI 5 was higher (33\%). However, among patients with lacunar syndrome, the prevalence of TIBI 5 was high, although their NIHSS were not low. A possible correlation between low NIHSS and high prevalence of TIBI 5 needs to be confirmed in larger studies. The treatment with intravenous thrombolysis 
Johansson et al.: Normal Findings on Pretreatment Transcranial Ultrasound in Patients Treated with Sonothrombolysis
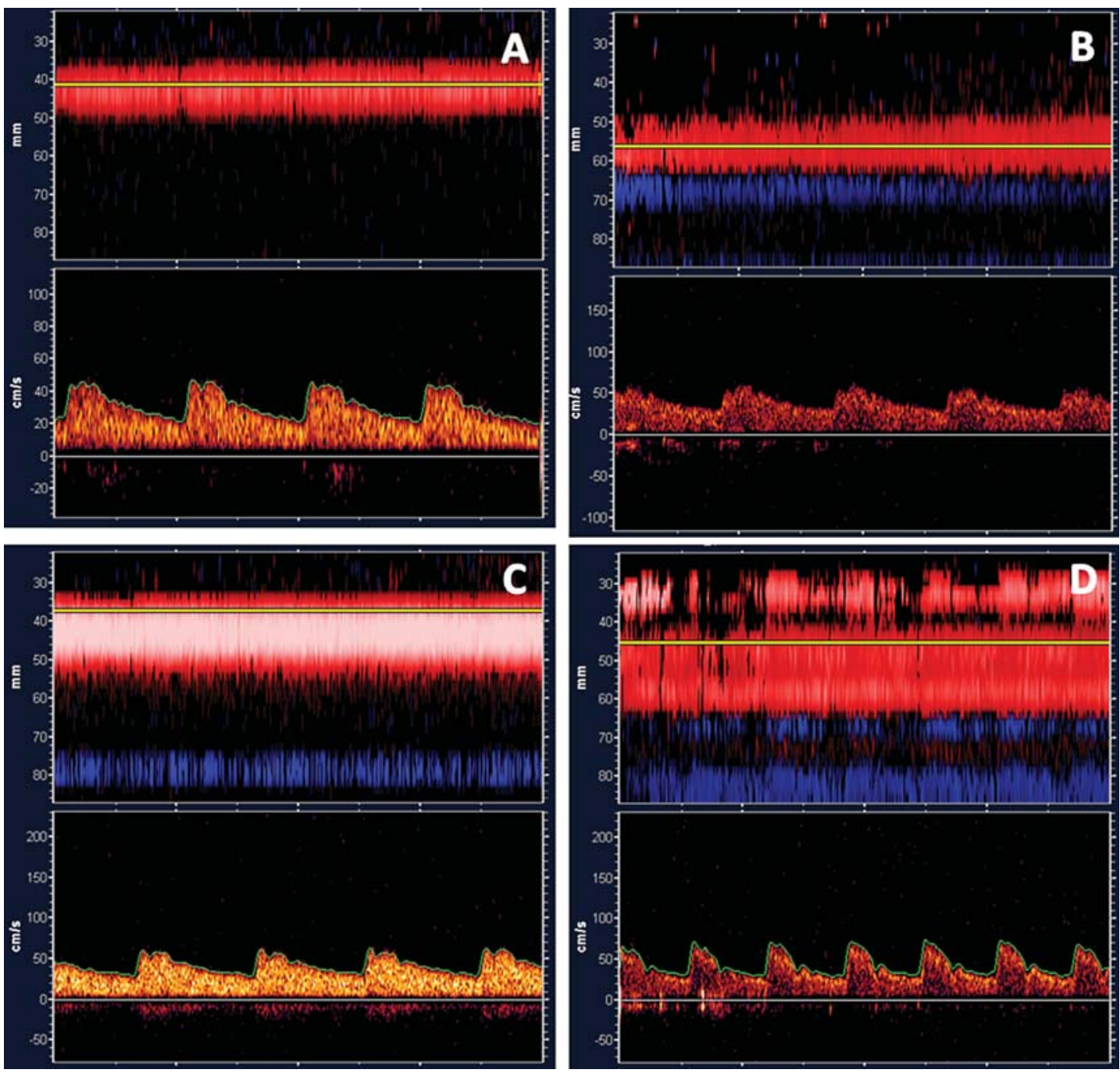

Fig. 1. TIBI 5 findings in cases 1-4 (for more details see table 1). A Case 1: the mean flow velocity was $12 \%$ higher compared to the contralateral side. B Case 2: the mean flow velocity was $5 \%$ lower compared to the contralateral side. C Case 3: the mean flow velocity was $15 \%$ lower compared to the contralateral side. D Case 4: the mean flow velocity was $35 \%$ higher compared to the contralateral side.

Table 2. Comparison of patients with TIBI 5 and TIBI $<5$ on pretreatment ultrasound examination

\begin{tabular}{llcc}
\hline & $\begin{array}{l}\text { TIBI }<5 \text { at baseline } \\
(\mathrm{n}=12)\end{array}$ & $\begin{array}{l}\text { TIBI } 5 \text { at baseline } \\
(\mathrm{n}=6)\end{array}$ & $\mathrm{p}$ value \\
\hline Median age (IQR), years & $74(67-79)$ & $62(40-77)$ & $0.19^{\mathrm{a}}$ \\
Male sex, $\mathrm{n}(\%)$ & $8(67)$ & $4(67)$ & $1.00^{\mathrm{b}}$ \\
Median NIHSS baseline (IQR) & $5(4-6)$ & $6(3-8)$ & $0.82^{\mathrm{a}}$ \\
NIHSS improved at discharge, $\mathrm{n}(\%)^{\mathrm{c}}$ & $9(75)$ & $2(50)$ & $0.35^{\mathrm{b}}$ \\
TOAST: lacunar syndrome, $\mathrm{n}(\%)^{\mathrm{c}}$ & $2(17)$ & $2(50)$ & $0.18^{\mathrm{b}}$ \\
\hline
\end{tabular}

${ }^{a}$ Mann-Whitney test. ${ }^{b} \chi^{2}$ test. $^{c}$ Two stroke mimics excluded. 
in patients with mild stroke (NIHSS $\leq 5$ ) is debated $[11,12]$. A reason for the low NIHSS and the high treatment rate $(11 \%)$ in this study is our selection policy for thrombolysis. At our stroke center, as well as in many other stroke units in Sweden and elsewhere, the decision of whether intravenous tPA is offered is made by the responsible stroke physician who takes into account the presumed long-term effects of the initial symptoms rather than the NIHSS itself.

Lacunar strokes have been associated with lower flow velocities in the posterior circulation compared to other etiologies 3-14 days after onset [13] and with increased (peak systolic velocity $>120 \mathrm{~cm} / \mathrm{s}$ or mean velocity $>80 \mathrm{~cm} / \mathrm{s}$ ) MCA flow in $45 \%$ of the patients within admission at day 5 [14]. However, studies in an acute setting (before administration of tPA) are lacking. Small deep cerebral infarcts that cause lacunar syndrome may be due to lipohyalinosis or occlusion of the parent artery [15]. In lipohyalinosis, it is likely that the flow in the main artery branches is left intact, and therefore, a normal finding on transcranial ultrasound is expected. This theory is strengthened by our observation of 1 case that experienced a temporary worsening during the tPA infusion, and this was neither associated with a change in MCA blood flow nor with an intracerebral hemorrhage. In cases with occlusion of the parent artery, a TIBI value $<5$ is expected - and this was detected in half of our cases with lacunar syndrome. Studies focusing on the association between TIBI grade at the initiation of thrombolysis treatment and stroke etiology are warranted.

The 2 patients with TIBI 5 and cortical syndrome both had partial symptom regression before treatment onset. Therefore, a likely explanation for the TIBI 5 finding in these cases is pretreatment recanalization. Since the NIHSS were low, another possible explanation is that a persistent artery occlusion in a small artery branch where the preocclusive flow changes (such as TIBI 3 in the entire M1) was masked by the normal flow to other branches. In the 2 cases of stroke mimic, TIBI 5 was an expected finding. What is noteworthy about these stroke mimic cases is that that they only comprised one third of patients treated with thrombolysis who had TIBI 5 at baseline. The other 4 patients with acute focal neurological deficits of presumed vascular origin and TIBI 5 had new cerebral infarctions on posttreatment CT scans, excluding the possibility that these patients were stroke mimics.

In patients with TIBI $0-4$, the ultrasound operator knows where to aim the ultrasound during sonothrombolysis. However, this is not the case in patients with TIBI 5, even if an artery occlusion exists. It is questionable whether sonothrombolysis has any treatment effects when the operator is likely to miss the artery occlusion. Therefore, due to the likely futility of sonothrombolysis under these conditions, some of the cases with TIBI 5 in this study were treated with thrombolysis alone. On the other hand, it is also likely that sonothrombolysis is not harmful for such patients compared to thrombolysis alone. An operator-independent sonothrombolysis device is being evaluated in the ongoing randomized trial CLOTBUST-ER [16]. This device should deliver ultrasound over a larger volume of tissue, which may theoretically be beneficial in cases with very distal thrombotic occlusion. However, it is unlikely that this can be evaluated in the ongoing trial because an NIHSS of $\geq 10$ is required for inclusion, whereas very distal occlusions are likely to have lower NIHSS. However, an additional randomized trial is ongoing that includes patients with TIBI 5 at treatment initiation [17].

This study has several weaknesses. It was based on our initial experiences, and therefore, our sample size was small. When managing the patients, we emphasized a short delay to treatment and TIBI grade changes during the treatment over detailed diagnostic descriptions. Therefore, we failed to gather data about the pulsatility index in various arterial segments and how the pulsatility index changed over time. 


\section{Conclusions}

In our experience, in patients with acute focal neurological deficits and indications for intravenous thrombolysis, it was not uncommon that the presumed affected artery was patent (TIBI 5) at the time of treatment onset. The likely pathophysiological mechanisms behind these findings were lacunar syndrome, pretreatment recanalization, and stroke mimic.

\section{Acknowledgements}

We would like to acknowledge the hard work of the staff of the Umeå Stroke Center. This study was financed with funds from the county of Västerbotten, the Swedish Heart and Lung Foundation, and the Northern Swedish Stroke Fund.

\section{Disclosure Statement}

The authors have no conflicts of interest to disclose.

\section{References}

1 Alexandrov AV, Sloan MA, Tegeler CH, Newell DN, Lumsden A, Garami Z, Levy CR, Wong LKS, Douville C, Kaps M, Tsivgoulis G; for the American Society of Neuroimaging Practice Guidelines Committee: Practice standards for transcranial Doppler (TCD) ultrasound. Part II. Clinical indications and expected outcomes. J Neuroimaging 2012;22:215-224.

$>2$ Tsivgoulis G, Alexandrov AV, Sloan MA: Advances in transcranial Doppler ultrasonography. Curr Neurol Neurosci Rep 2009;9:46-54.

-3 Alexandrov AV, Molina CA, Grotta JC, Garami Z, Ford SR, Alvarez-Sabin J, Montaner J, Saqqur M, Demchuk AM, Moye LA, Hill MD, Wojner AW; CLOTBUST Investigators: Ultrasound-enhanced systemic thrombolysis for acute ischemic stroke. N Engl J Med 2004;351:2170-2178.

4 Demchuk AM, Burgin WS, Christou I, Felberg RA, Barber PA, Hill MD, Alexandrov AV: Thrombolysis in brain ischemia (TIBI) transcranial Doppler flow grades predict clinical severity, early recovery, and mortality in patients treated with intravenous tissue plasminogen activator. Stroke 2001;32:89-93.

-5 Chernyshev OY, Garami Z, Calleja S, Song J, Campbell MS, Noser EA, Shaltoni H, Chen CI, Iguchi Y, Grotta JC, Alexandrov AV: Yield and accuracy of urgent combined carotid/transcranial ultrasound testing in acute cerebral ischemia. Stroke 2005;36:32-37.

6 Tsivgoulis G, Eggers J, Ribo M, Perren F, Saqqur M, Rubiera M, Sergentanis TN, Vadikolias K, Larrue V, Molina CA, Alexandrov AV: Safety and efficacy of ultrasound-enhanced thrombolysis: a comprehensive review and meta-analysis of randomized and nonrandomized studies. Stroke 2010;41:280-387.

7 Alexandrov AV: Cerebrovascular Ultrasound in Stroke Prevention and Treatment, ed 2. West Sussex, John Wiley \& Sons Ltd, 2011.

8 Adams HP Jr, Bendixen BH, Kappelle LJ, Biller J, Love BB, Gordon DL, Marsh EE 3rd; the TOAST Investigators: Classification of subtype of acute ischemic stroke definitions for use in a multicenter clinical trial. Stroke 1993; 24:35-41.

-9 Tsivgoulis G, Sharma VK, Lao AY, Malkoff MD, Alexandrov AV: Validation of transcranial Doppler with computed tomography angiography in acute cerebral ischemia. Stroke 2007;38:1245-1249.

10 Tsivgoulis G, Ribo M, Rubiera M, Vasdekis SN, Barlinn K, Athanasiadis D, Shahripour BR, Giannopoulos S, Stamboulis E, Harrigan MR, Molina CA, Alexandrov AV: Real-time validation of transcranial Doppler criteria in assessing recanalization during intra-arterial procedures for acute ischemic stroke: an international, multicenter study. Stroke 2013;44:394-400.

-11 Urra X, Ariño H, Llull L, Amaro S, Obach V, Cervera Á, Chamorro Á: The outcome of patients with mild stroke improves after treatment with systemic thrombolysis. PLoS One 2013;8:e59420.

12 Strbian D, Piironen K, Meretoja A, Sairanen T, Putaala J, Tiainen M, Artto V, Rantanen K, Häppölä O, Kaste M, Lindsberg PJ; for the Helsinki Stroke Thrombolysis Registry Group: Intravenous thrombolysis for acute ischemic stroke patients presenting with mild symptoms. Int J Stroke 2013;8:293-299. 
13 Kim JT, Lee SH, Hur N, Jeong SK: Blood flow velocities of cerebral arteries in lacunar infarction and other ischemic strokes. J Neurol Sci 2011;308:57-61.

14 Gujjar AR, William R, Jacob PC, Jain R, Al-Asmi AR: Transcranial Doppler ultrasonography in acute ischemic stroke predicts stroke subtype and clinical outcome: a study in Omani population. J Clin Monitor Comput 2011; 25:121-128.

15 Del Bene A, Palumbo V, Lamassa M, Saia V, Piccardi B, Inzitari D: Progressive lacunar stroke: review of mechanisms, prognostic features, and putative treatments. Int J Stroke 2012;7:321-329.

16 The CLOBUST-ER trial. http://www.clinicaltrials.gov NCT01098981.

17 The NOR-SASS trial. http://www.clinicaltrials.gov_NCT01949961. 